\title{
Using Sandwich Composite Shells for Fully Pressurized Tanks on Liquefied Petroleum Gas Carriers
}

\author{
Veysel Alankaya ${ }^{1, *}$ - Fuat Alarçin² \\ 1Turkish Naval Academy, Department of Naval Architecture, Turkey \\ ${ }^{2}$ Y 1 ldız Technical University, Department of Marine Engineering Operation, Turkey
}

\begin{abstract}
The growth of shipping as the main link of the seaborne gas supply chain results from the growing demand for liquefied petroleum gasses due to their various uses, such as fuel for cooking/heating, automotive power, and numerous applications. This study investigates the structural suitability of sandwich composite materials for pressurized tanks, which can be used in on-board carriers or barges for transportation, as well as inland for industrial or residential storage needs. The analytical solution methodology for analysing stresses and deformations is based on higher order shear deformation theory (HSDT). A boundary discontinuous generalized double Fourier series approach is used to solve highly coupled linear partial differential equations. The complementary boundary constraints are introduced through boundary discontinuities, generated by the selected boundary conditions for the derivation of the complementary solution. Numerical solutions are presented for laminated sandwich shells having both cylindrical and spherical forms, which are the preferred geometries for pressurized tanks.
\end{abstract}

Keywords: sandwich composites, pressurized tanks, boundary-discontinuity, doubly curved shell, liquefied petroleum gas carriers

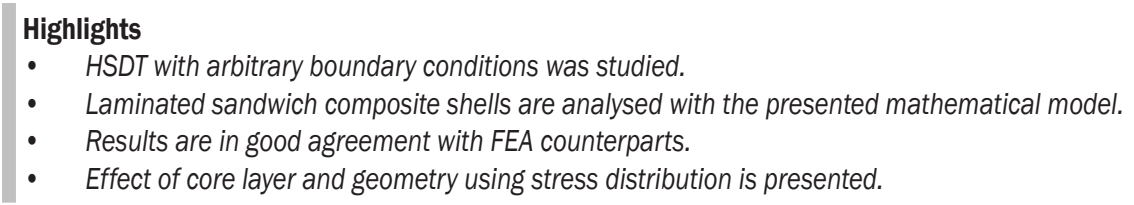

\section{INTRODUCTION}

Petroleum gases, an alternative to fuel oil with lower prices, are preferred for numerous uses, which can be briefly listed as (i) in-house basic needs, such as cooking and heating, (ii) industrial needs for power plants, plastic and chemical applications, and (iii) propulsion fuel for transportation. This large operational suitability causes the configuration of a distribution network depending on pipelines for the areas with infrastructure, storages for field usage, or shipping solutions for seaborne supply.

The U.S. Energy Administration estimates energy consumption, which was approximately 825 million barrels in 2013, to increase by $0.6 \%$ annually from 2012 to 2040 while the natural oil resources are declining [1].

Since many world oil resources are difficult to reach, growth in sea gas transport capacity and the construction of new gas transport pipelines are expected.

As indicated by Fujitani et al. [2], while pipelines on land and in the sea can deliver the gas in gaseous form only over relatively short distances, another means of transporting the gas in larger quantities over longer distances is required.

The most cost efficient way of transporting gas between continents is to carry it in liquid form using ships. Further transport cost reductions can be achieved by decreasing the weight of the ship using composite materials rather than steel, since composites have a higher weight-to-stiffness ratio.

The aim of this study is to investigate the suitability of sandwich composite shells as structural members of cylindrical or spherical tanks by analysing stress distribution through the shell thickness and the shell deformations under pressure loading.

\section{PRESSURIZED LIQUEFIED GAS TANKS}

Liquefied gasses are defined as consisting of a broad range of petroleum gas mixtures, which, as listed in the rules of Turkish Lloyd [3], include: acetaldehyde, ammonia, butane, carbon dioxide, ethane, ethylene, nitrogen, refrigerant gasses, sulphur dioxide, etc.

Liquefied gas transportation distinguishes three major gas conditions: (i) fully pressurized (pressurized at ambient temperature), (ii) semi-refrigerated (pressurized and refrigerated at optimum temperature and pressure), (iii) fully refrigerated (refrigerated at or near atmospheric pressure). The loads associated with these liquefied gas conditions are one of the main characteristics that determine the pressurized tank design. For refrigerated tanks, the tank material's lowtemperature toughness becomes the most important property to consider, since most materials become 
brittle below a certain temperature. Some liquefied gasses, such as ammonia, butane, propane, propylene and vinyl chloride, can be transported at ambient temperatures. Although the storage temperature is not considered for liquefied gasses, operating temperature caused by environmental conditions shall be taken into account in terms of stationary thermal loads. The operating temperature may give rise to significant thermal stresses. Therefore, each cargo tank is fitted with at least one liquid level gauging device, designed to operate at pressures not less than the maximum allowable relief valve setting at the temperatures within the cargo operating temperature range [3].

These liquefied gasses are transported via cylindrical or spherical cargo tanks at a vapour pressure of maximum $18 \mathrm{MPa}$, as indicated by Fujitani et al. [2]. In this study, the main loading is considered to be the maximum vapour pressure, ignoring thermal stresses caused by operating temperature and environmental conditions.

In addition to the liquefied gas storage temperature, tank design is also done according to the cargo tank arrangement restrictions from the International Code for the construction and equipment of ship carrying liquefied gasses in bulk (ICG code), incorporated into the Safety of life at sea (SOLAS) convention. According to the ICG code, liquefied gasses that do not need to be refrigerated, are restricted to Type $\mathrm{C}$ tanks, which are structurally independent of the ship's hull. Fig. 1 shows a generic mid-ship section view of a hull and cargo tank.

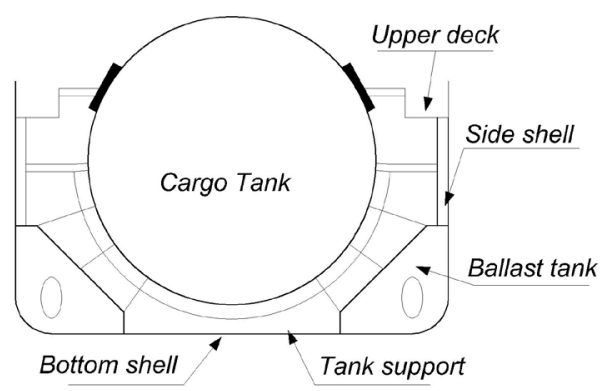

Fig. 1. Mid-ship section of the hull and cargo tank

Type C cargo tanks are completely selfsupporting and do not form part of the ship's hull, nor do they contribute to the hull strength [2]. Similar applications of independent tanks are also common at liquefied natural gas (LNG) ships, as presented in Fig. 2. However LNG tanks, which store the gas at $-162{ }^{\circ} \mathrm{C}$, are not investigated in this study, because the refrigeration temperature effect on the tank material is beyond the scope of this study. Nevertheless, since
LNG tanks are cylindrical and spherical in shape, the present methodology also could be applied to these tanks.

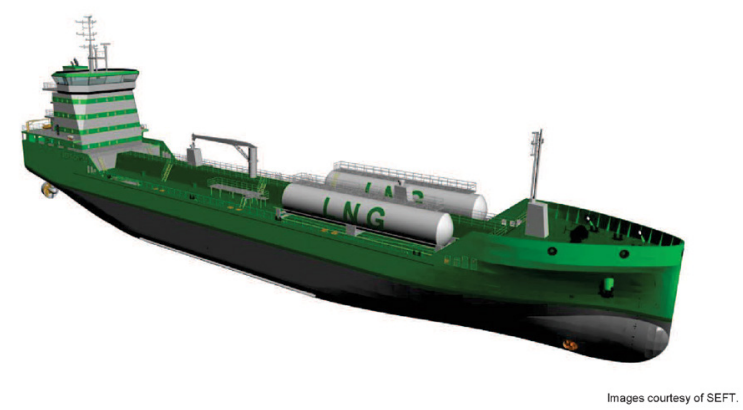

Fig. 2. Hull-independent tank on an LNG carrier [4]

\section{COMPOSITE MATERIALS}

As presented by Mouritz et al. [5], composite materials can be chosen for several functional components of ships, such as hull (complete or partial as for bulkheads), superstructure (complete for small crafts, or partial for bigger crafts), for the mast, for the propulsion system components, and even for propellers.

One major reason for these choices is the composites' high stiffness-to-weight ratio, which reduces the components weight; another reason is the lower cost of moulding complex geometry parts rather than machining them.

Moreover, the design flexibility inherent in composite laminates, which is described as tailoring by Kabir et al. [6], makes composites a reasonable choice for obtaining optimum specific design requirements through a combination of structural/ material concepts, stacking sequence, ply orientation, choice of the component phases, etc. These allow composites to provide modern solutions for thermal and acoustic insulation, stealth capabilities, shock resistance, etc.

When Liquefied Petroleum Gas (LPG) is in a hull-independent type $\mathrm{C}$ tank, no thermal insulation is required. Therefore, the design of the material can be tailored to be non-flammable and to bear the load while minimizing weight.

Utilization of composite materials, however, also brings difficulties to the analyst, such as the interlaminar or transverse shear stress due to mismatch of material properties among layers, bendingstretching coupling due to lamination asymmetry, and in-plane orthotropy. The transverse stress and strain components are ignored in classical or thin shell theories, which makes these theories inadequate 
for the analysis of thicker shells. Hence, reliable prediction of deformations and stresses for thicker structures require the use of higher order shear deformation theories, which are based on a cubic or higher expansion of the in-plane displacements. Higher order theories introduce additional unknowns that are difficult to interpret in physical terms and require more mathematical computations for finding solutions [7] and [8].

As indicated by Youssif [9], to analyse the effects of design sensitivities efficiently and accurately, it is crucial to have the appropriate techniques associated with good structural models. Therefore, it is essential to develop a solution methodology considering the additional complexities arising by way of satisfying boundary conditions that cannot be handled by Navier's or Levy's traditional analytical approaches. Chaudhuri [10] provides the mathematical explanations for the boundary discontinuous type Fourier series approach for solving completely coupled system of partial differential equations, subjected to admissible general boundary conditions.

In this particular study, static deflections of cylindrical and spherical shaped pressurized tanks made of sandwich composites are investigated by using the higher order shear deformation theory. The effects of boundary conditions on the solution functions are introduced as described by Oktem and Chaudhuri [11] and the presented solution methodology is developed to apply to sandwich composites.

\section{DEFINITION OF THE PROBLEM}

The geometry of a composite shell, which consists of laminated plies with uniform thicknesses is shown in Fig. 3, where $a$ and $b$ represent the dimensions in the $\xi_{1}, \xi_{2}$ axes respectively, while $\xi_{3}$ is a line normal to the mid-surface defined at the centre going through the shell thickness $h$. The terms $R_{1}$ and $R_{2}$ are the midsurface curvatures in the $\left(\xi_{1}, \xi_{2}\right)$ axes respectively.

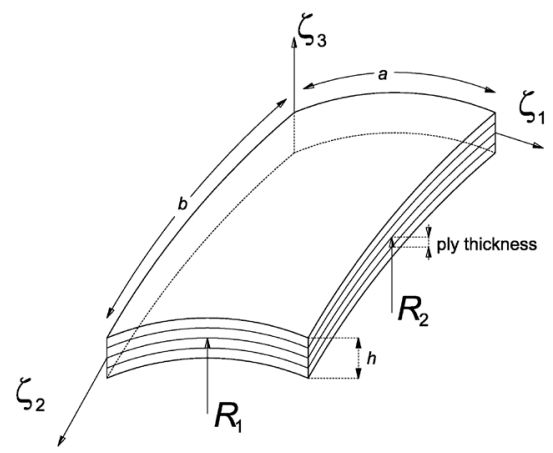

Fig. 3. Rectangular panel geometry of a composite shell
Fig. 4 shows the ply distance $\mathrm{z}$ from the midsurface. The terms $\phi_{1}$ and $\phi_{2}$ are rotations about $\xi_{2}$ and $\xi_{1}$ axes, respectively. Details of the straindisplacement relations and other explanations are given by Reddy [12].

a)

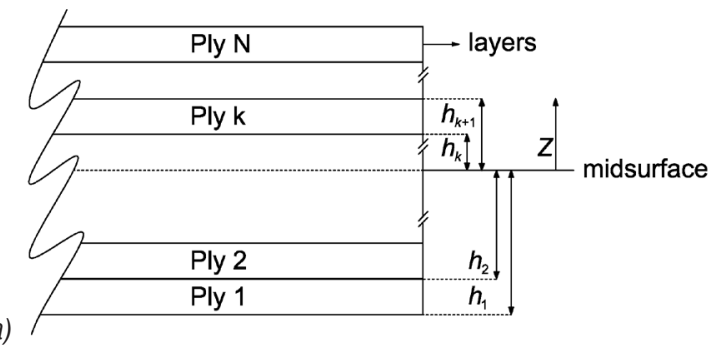

b)

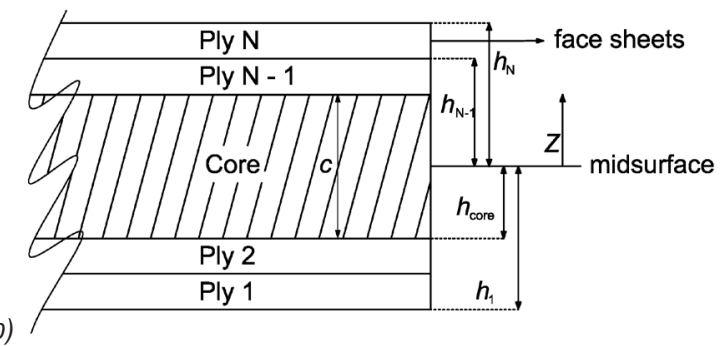

Fig. 4. Ply distances from the mid-surface for a laminated shell; a)without a core, and b) with a sandwich core

The displacement field by considering the cubic terms and satisfying the conditions of transverse shear stresses (and hence strains) vanishing at a point $\left(\xi_{1}, \xi_{2}, \pm h / 2\right)$ on the top and bottom surfaces of the shell, is given by Reddy [12] as follows:

$$
\begin{aligned}
& u_{1}=u\left(\xi_{1}, \xi_{2}, \xi_{3}\right)=u_{0}\left(\xi_{1}, \xi_{2}\right)+ \\
&+z \varphi_{1}\left(\xi_{1}, \xi_{2}\right)-\frac{4}{3 h^{2}} z^{3}\left(\varphi_{1}+\frac{\partial w_{0}}{\partial \xi_{1}}\right), \\
& u_{2}= v\left(\xi_{1}, \xi_{2}, \xi_{3}\right)=v_{0}\left(\xi_{1}, \xi_{2}\right)+ \\
&+z \varphi_{2}\left(\xi_{1}, \xi_{2}\right)-\frac{4}{3 h^{2}} z^{3}\left(\varphi_{2}+\frac{\partial w_{0}}{\partial \xi_{2}}\right), \\
& u_{3}=w\left(\xi_{1}, \xi_{2}, \xi_{3}\right)=w_{0}\left(\xi_{1}, \xi_{2}\right),
\end{aligned}
$$

where $u, v, w$ represent displacements of a point at three axes $\xi_{1}, \xi_{2}, \xi_{3}$, while $u_{0}, v_{0}, w_{0}$ are displacements of a point at the mid-surface $\left(\xi_{3}=0\right)$.

Equilibrium equations derived by using the principles of virtual work are given as follows [12]:

$$
\begin{aligned}
& \frac{\partial N_{1}}{\partial \xi_{1}}+\frac{\partial N_{6}}{\partial \xi_{2}}=0, \\
& \frac{\partial N_{6}}{\partial \xi_{1}}+\frac{\partial N_{2}}{\partial \xi_{2}}=0,
\end{aligned}
$$




$$
\begin{gathered}
\frac{\partial Q_{1}}{\partial \xi_{1}}+\frac{\partial Q_{2}}{\partial \xi_{2}}-\frac{4}{h^{2}}\left(\frac{\partial K_{1}}{\partial \xi_{1}}+\frac{\partial K_{2}}{\partial \xi_{2}}\right)+ \\
+\frac{4}{3 h^{2}}\left(\frac{\partial^{2} P_{1}}{\partial \xi_{1}^{2}}+\frac{\partial^{2} P_{2}}{\partial \xi_{2}^{2}}+2 \frac{\partial^{2} P_{6}}{\partial \xi_{1} \partial \xi_{2}}\right)=-q \\
\frac{\partial M_{1}}{\partial \xi_{1}}+\frac{\partial M_{6}}{\partial \xi_{2}}-Q_{1}+\frac{4}{h^{2}} K_{1}-\frac{4}{3 h^{2}}\left(\frac{\partial P_{1}}{\partial \xi_{1}}+\frac{\partial P_{6}}{\partial \xi_{2}}\right)=0, \\
\frac{\partial M_{6}}{\partial \xi_{1}}+\frac{\partial M_{2}}{\partial \xi_{2}}-Q_{2}+\frac{4}{h^{2}} K_{2}-\frac{4}{3 h^{2}}\left(\frac{\partial P_{6}}{\partial \xi_{1}}+\frac{\partial P_{2}}{\partial \xi_{2}}\right)=0 .
\end{gathered}
$$

In Eq. (2), $q$ is the transverse load and $N_{i}, M_{i}$, $\left.P_{i}, i=1,2,6\right)$ denotes stress resultants, stress couples and second stress couples (see, e.g., Reddy [12]). $Q_{i}(i=1,2)$ represents the transverse shear stress resultants. They are given as follows:

$$
\begin{gathered}
N_{i}=A_{i j} \varepsilon_{j}^{0}+B_{i j} \kappa_{j}^{0}+E_{i j} \kappa_{j}^{2}, \quad(i, j=1,2,6), \\
M_{i}=B_{i j} \varepsilon_{j}^{0}+D_{i j} \kappa_{j}^{0}+F_{i j} \kappa_{j}^{2}, \\
P_{i}=E_{i j} \varepsilon_{j}^{0}+F_{i j} \kappa_{j}^{0}+H_{i j} \kappa_{j}^{2}, \\
Q_{1}=A_{5 j} \varepsilon_{j}^{0}+D_{5 j} \kappa_{j}^{1}, \quad(j=4,5), \\
Q_{2}=A_{4 j} \varepsilon_{j}^{0}+D_{4 j} \kappa_{j}^{1}, \\
K_{1}=D_{5 j} \varepsilon_{j}^{0}+F_{5 j} \kappa_{j}^{1}, \\
K_{2}=D_{4 j} \varepsilon_{j}^{0}+F_{4 j} \kappa_{j}^{1},
\end{gathered}
$$

in which $A_{i j}, B_{i j}$, etc. are the laminate rigidities (integrated stiffnesses). These are given as follows:

$$
\begin{gathered}
\left(A_{i j}, B_{i j}, D_{i j}\right)=\sum_{k=1}^{N} \int_{\xi_{k-1}}^{\xi_{k}} Q_{i j}^{(k)}\left(1, z, z^{2}\right) d z, \\
\left(E_{i j}, F_{i j}, H_{i j}\right)=\sum_{k=1}^{N} \int_{\xi_{k-1}}^{\xi_{k}} Q_{i j}^{(k)}\left(z^{3}, z^{4}, z^{6}\right) d z .
\end{gathered}
$$

Generalized stress-strain constitutive relations for an orthogonal lamina can be expressed as follows:

$$
\left\{\begin{array}{l}
\sigma_{1} \\
\sigma_{2} \\
\sigma_{6} \\
\sigma_{5} \\
\sigma_{4}
\end{array}\right\}=\left[\begin{array}{ccccc}
Q_{11} & Q_{12} & 0 & 0 & 0 \\
Q_{12} & Q_{22} & 0 & 0 & 0 \\
0 & 0 & Q_{66} & 0 & 0 \\
0 & 0 & 0 & Q_{55} & 0 \\
0 & 0 & 0 & 0 & Q_{44}
\end{array}\right]\left\{\begin{array}{c}
\varepsilon_{1} \\
\varepsilon_{2} \\
\varepsilon_{6} \\
\varepsilon_{5} \\
\varepsilon_{4}
\end{array}\right\},
$$

in which $\sigma_{1}, \sigma_{2}, \sigma_{6}, \sigma_{5}, \sigma_{4}$ are the stress and $\varepsilon_{1}, \varepsilon_{2}, \varepsilon_{6}, \varepsilon_{5}, \varepsilon_{4}$ are the strain components. $Q_{i j}$ expressions in terms of engineering constants are given below:

$$
\begin{gathered}
Q_{11}=\frac{E_{1}}{1-v_{12} v_{21}}, \quad \text { (6b) } Q_{12}=\frac{v_{12} E_{2}}{1-v_{12} v_{21}}, \\
Q_{22}=\frac{E_{2}}{1-v_{12} v_{21}}, \quad \text { (6d) } \quad Q_{66}=G_{12}, \\
Q_{44}=G_{23}, \quad(6 \mathrm{f}) \quad Q_{55}=G_{13} .
\end{gathered}
$$

The introduction of Eqs. (3) and (4) into Eq. (2) gives five highly coupled fourth-order partial differential equations. The set of equations can be expressed in the following form:

$$
K_{i j} x_{j}=f_{i} \quad(i, j=1, \ldots, 5) \text { and }\left(K_{i j}=K_{j i}\right),
$$

where

$$
\begin{aligned}
& \left\{x_{j}\right\}^{T}=\left\{\begin{array}{lllll}
u_{1} & u_{2} & u_{3} & \phi_{1} & \phi_{2}
\end{array}\right\}, \\
& \left\{f_{i}\right\}^{T}=\left\{\begin{array}{llllll}
0 & 0 & -Q_{m n} & 0 & 0
\end{array}\right\} .
\end{aligned}
$$

Definitions of $\left[K_{i j}\right]$ elements are given in Appendix A. The load term $\left(Q_{m n}\right)$ is a uniformly distributed load (the main load definition for pressurized tanks), and is defined as [11]:

$$
Q_{m n}=\frac{16 q}{\pi^{2} m n}
$$

\section{SOLUTION METHODOLOGY}

A tank surface composed of cylindrical and spherical shells can simply be considered to be a doubly curved shell. If part of the tank shell, in the form of a rectangular panel, is inspected separately, the orthotropic shell cannot move in the longitudinal or transversal directions. However, rotations on the edges are possible. It is assumed that these constraints ensure that the following boundary conditions are true for all the edges of the selected shell part.

$$
\begin{aligned}
& u_{3}\left(0, \xi_{2}\right)=u_{3}\left(a, \xi_{2}\right)=u_{3}\left(\xi_{1}, 0\right)=u_{3}\left(\xi_{1}, b\right)=0 \\
& u_{1}\left(0, \xi_{2}\right)=u_{1}\left(a, \xi_{2}\right)=u_{1}\left(\xi_{1}, 0\right)=u_{1}\left(\xi_{1}, b\right)=0 \\
& u_{2}\left(0, \xi_{2}\right)=u_{2}\left(a, \xi_{2}\right)=u_{2}\left(\xi_{1}, 0\right)=u_{2}\left(\xi_{1}, b\right)=0 \\
& \phi_{1}\left(\xi_{1}, 0\right)=\phi_{1}\left(\xi_{1}, b\right)=\phi_{2}\left(0, \xi_{2}\right)=\phi_{2}\left(b, \xi_{2}\right)=0 \\
& M_{2}\left(\xi_{1}, 0\right)=M_{2}\left(\xi_{1}, b\right)=M_{1}\left(0, \xi_{2}\right)=M_{1}\left(b, \xi_{2}\right)=0
\end{aligned}
$$


The particular solution to the boundary-value problem of an HSDT-based cross-ply shell assumes amplitudes $U_{m n}, V_{m n}$ and $W_{m n}$ at $\xi_{1}, \xi_{2}, \xi_{3}$ axes, respectively and $X_{m n}, Y_{m n}$ for rotations about $\xi_{2}$ and $\xi_{1}$ axes. Solution functions are examined at the shell boundaries through the length $a$ and width $b$ of the shell geometry and organized solution functions at the boundaries are settled by equalities [12].

$$
\begin{array}{cl}
u_{1}\left(\xi_{1}, \xi_{2}\right)=\sum_{m=0}^{\infty} \sum_{n=1}^{\infty} U_{m n} \Re_{1}\left(\xi_{1}, \xi_{2}\right), & 0<\xi_{1}<a, \\
0 \leq \xi_{2} \leq b, \\
u_{2}\left(\xi_{1}, \xi_{2}\right)=\sum_{m=1}^{\infty} \sum_{n=0}^{\infty} V_{m n} \Re_{2}\left(\xi_{1}, \xi_{2}\right), & 0 \leq \xi_{1} \leq a, \\
& 0<\xi_{2}<b, \\
u_{3}\left(\xi_{1}, \xi_{2}\right)=\sum_{m=1}^{\infty} \sum_{n=1}^{\infty} W_{m n} \Re_{3}\left(\xi_{1}, \xi_{2}\right), & 0 \leq \xi_{1} \leq a, \\
& 0 \leq \xi_{2} \leq b, \\
\varphi_{1}\left(\xi_{1}, \xi_{2}\right)=\sum_{m=0}^{\infty} \sum_{n=1}^{\infty} X_{m n} \Re_{1}\left(\xi_{1}, \xi_{2}\right), & 0 \leq \xi_{1} \leq a, \\
& 0 \leq \xi_{2} \leq b, \\
\varphi_{2}\left(\xi_{1}, \xi_{2}\right)=\sum_{m=1}^{\infty} \sum_{n=0}^{\infty} Y_{m n} \Re_{2}\left(\xi_{1}, \xi_{2}\right), & 0 \leq \xi_{1} \leq a, \\
& 0 \leq \xi_{2} \leq b,
\end{array}
$$

where,

$$
\begin{gathered}
\Re_{1}\left(\xi_{1}, \xi_{2}\right)=\cos \left(\alpha \xi_{1}\right) \sin \left(\beta \xi_{2}\right), \\
\Re_{2}\left(\xi_{1}, \xi_{2}\right)=\sin \left(\alpha \xi_{1}\right) \cos \left(\beta \xi_{2}\right), \\
\Re_{3}\left(\xi_{1}, \xi_{2}\right)=\sin \left(\alpha \xi_{1}\right) \sin \left(\beta \xi_{2}\right), \\
\alpha=\frac{m \pi}{a}, \quad \beta=\frac{n \pi}{b} .
\end{gathered}
$$

The next step is substituting assumed particular solutions (Eq. (10)) into equilibrium Eq. (2). The differentiation procedure for these functions is based on Lebesque integration theory, which introduces boundary Fourier coefficients arising from discontinuities of the particular solutions at the edges. As has been noted by Chaudhuri [10], the boundary Fourier coefficients serve as a complementary solution to the problem under investigation. The partial derivatives, which cannot be obtained by term-wise differentiation, are given by Oktem and Chaudhuri [11], as follows,

$$
u_{1,1}=-\sum_{m=1}^{\infty} \sum_{n=1}^{\infty} \alpha U_{m n} \sin \left(\alpha \xi_{1}\right) \sin \left(\beta \xi_{2}\right)
$$

$$
\begin{aligned}
& u_{1,11}=\frac{1}{2} \sum_{m=1}^{\infty} \overline{c_{m}} \sin \left(\beta \xi_{2}\right)+ \\
& +\sum_{m=1}^{\infty} \sum_{n=1}^{\infty}\left\{-\alpha^{2} U_{m n}+\overline{a_{n}} \gamma_{m}+\overline{b_{n}} \delta_{m}\right\} \sin \left(\alpha \xi_{1}\right) \cos \left(\beta \xi_{2}\right),(13) \\
& u_{1,111}=-\sum_{m=1}^{\infty} \sum_{n=1}^{\infty} \alpha\left\{-\alpha^{2} U_{m n}+\overline{a_{n}} \gamma_{m}+\overline{b_{n}} \delta_{m}\right\} \times \\
& \times \sin \left(\alpha \xi_{1}\right) \sin \left(\beta \xi_{2}\right) \\
& u_{2,2}=-\sum_{m=1}^{\infty} \sum_{n=1}^{\infty} \beta V_{m n} \sin \left(\alpha \xi_{1}\right) \sin \left(\beta \xi_{2}\right) \\
& u_{2,22}=\frac{1}{2} \sum_{m=1}^{\infty} \overline{c_{m}} \sin \left(\alpha \xi_{2}\right)+ \\
& +\sum_{m=1}^{\infty} \sum_{n=1}^{\infty}\left\{-\beta^{2} V_{m n}+\bar{c}_{m} \gamma_{n}+\overline{d_{m}} \delta_{n}\right\} \sin \left(\alpha \xi_{1}\right) \cos \left(\beta \xi_{2}\right) .(16)
\end{aligned}
$$

The boundary Fourier coefficients $\left(\overline{a_{n}}, \overline{b_{n}}\right)$ etc., which appear in Eqs. (8) to (12), are defined in Appendix B. The remaining partial derivatives can be obtained by term-wise differentiation.

The resulting equations are supplied by the geometric and natural boundary conditions, which are presented by Oktem and Chaudhuri [11], and are not rewritten here to keep the presentation shorter.

\section{NUMERICAL RESULTS AND DISCUSSIONS}

Numerical results are presented for composites, for which laminates have a cross-ply $\left[0^{\circ} / 90^{\circ}\right]$ arranged carbon fibres impregnated in an epoxy resin prepreg, with the polyurethane foam core material of varying thicknesses. The doubly-curved shell geometry includes cylindrical and spherical tank forms. The applied loading is a pressure with a value of $18 \mathrm{MPa}$, which is the maximum storage pressure for LPG carriers [2].

For the numerical solutions, commercially available materials are chosen. The following material properties are provided from their producers. Carbon epoxy prepreg is used for face sheets and generic polyurethane foam for core layers.

Table 1. Material properties of the face sheets and the core

\begin{tabular}{lcc}
\hline Part & Material & Property \\
\hline Face Sheets & $\begin{array}{c}\text { Carbon Epoxy } \\
\left(\mathrm{AS} 4 / 3501-6\left[0^{\circ} / 90^{\circ}\right] \mathrm{s}\right)\end{array}$ & $\begin{array}{c}E=68.3 \mathrm{GPa}, \nu=0.05 \\
\left(\text { at } 121^{\circ} \mathrm{C}\right)\end{array}$ \\
\hline Core & Polyurethane foam & $\begin{array}{r}E=225 \mathrm{MPa}, \mathrm{G}=67.2 \mathrm{MPa} \\
\left(\text { at } 70^{\circ} \mathrm{C}\right)\end{array}$ \\
\hline
\end{tabular}


Here $E$ is the in-plane Young's modulus, and $G$ denotes in-plane shear modulus while $v$ is major Poisson's ratio.

In the calculations, the following normalized quantities are defined:

$$
\begin{gathered}
u_{3}^{*}=\left(10^{3} E h^{3} / p_{0} a^{4}\right) u_{3}, \\
M_{1}^{*}=\left(10^{3} / p_{0} a^{2}\right) M_{1}, \\
\sigma_{1}^{*}=10\left(h^{2} / p_{0} b^{2}\right) \sigma_{1},
\end{gathered}
$$

in which $a$ and $b$ are the edge length values of the panel, and $p_{0}$ denotes the transverse load. For the numerical results presented in the tables and figures, the normalized quantities are computed at the centre of the panel.

Varying values of shallowness ratio $R / L$ (radius/length) and thickness ratio $a / h$ (edgelength/thickness) are considered to show the effect of geometry changes. In addition, the effect of the core thickness is investigated by means of stress distribution through the thickness of the shell.

The solution methodology requires the control of convergence in order to define the required number of terms to include in the equations. Fig. 5 shows the convergence of the normalized central transverse displacement $u_{3}^{*}$ and the moment $M_{1}^{*}$ of a moderately thick $a / h=10$ symmetric cross-ply $\left[0^{\circ} / 90^{\circ}\right]_{\mathrm{s}}$ sandwich shell, having a core thickness $c$ ratio $a / c=10$ under a pressure loading of $18 \mathrm{MPa}$. The normalized displacement $u_{3}^{*}$ and moment $M_{1}^{*}$ exhibit a fast convergence. Nonetheless, the selected number of terms to be used in the numerical analyses is $n=m=40$.

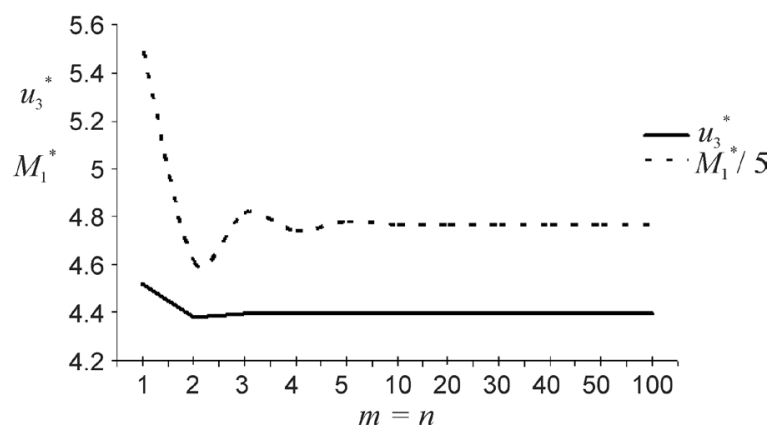

Fig. 5. Convergence of the normalized central deflection $u_{3}^{*}$ and moment $M_{1}^{*}$ for a symmetric moderately thick cross-ply $\left[0^{\circ} / 90^{\circ}\right]_{s}$ sandwich shell

The obtained results are compared to the results of the counterpart analyses using the first order shear deformation theory (FSDT) and the finite element analyses (FEA). The computer program used for validation purposes is the commercially available FEA software ANSYS. Shell geometry is modeled with $R / L=10$ shallowness at both radii $R_{1}$ and $R_{2}$, and with the ANSYS shell 91 element type with the sandwich option activated. Uniformly distributed loading is applied as pressure loading over the shell surface. The FEA layered shell model is presented in Fig. 6.

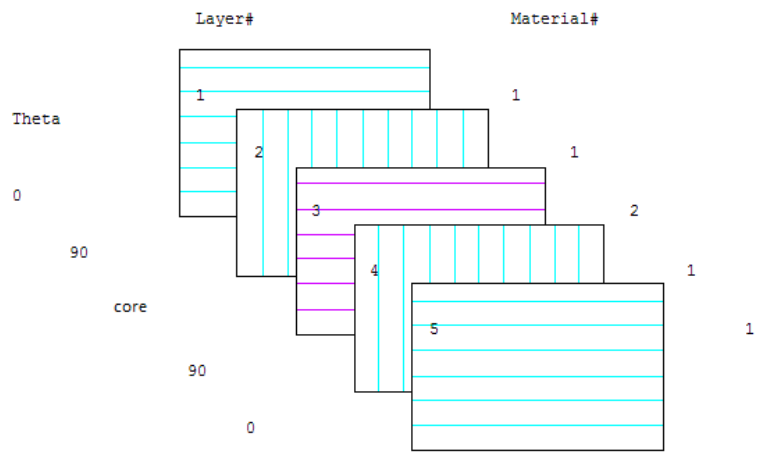

Fig. 6. Layered element model for a $0^{\circ} / 90^{\circ} /$ core $/ 90^{\circ} / 0^{\circ}$ laminate

The results of the present theory are in concordance with FEA and FSDT counterparts, as can be seen in Fig. 7, which compares varying $a / h$ ratios by means of the normalized deflection $u_{3}^{*}$ at the centre of the shell for the core material thickness ratio $a / c$ of 10 and 100.

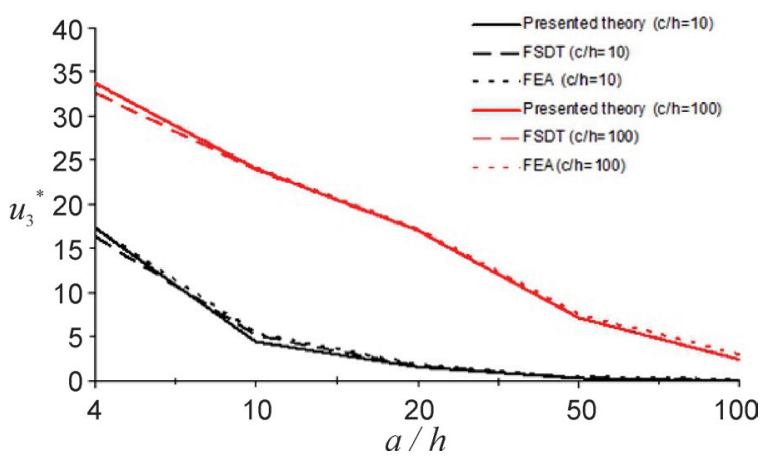

Fig. 7. Results from the present theory compared to the FEA and FSDT counterparts for varying a $/ h$ ratios by means of the normalized deflection $u_{3}^{*}$ at the centre of the shell

The effect of changing the panel dimensions is investigated by analysing the deflection and moment values at the centre of the shell. The results are presented in Fig. 8 according to varying ratios of edge lengths $a / b$ where increasing in this ratio causes the panel to become a beam. Major differences arise as a result of the effect of core material addition that was analyzed through central normalized deflections. Therefore, sandwich shells are suitable not only because they are stiff, but also because they can be 
tailored to additional requirements, such as thermal insulation or fire retardancy of the core material.

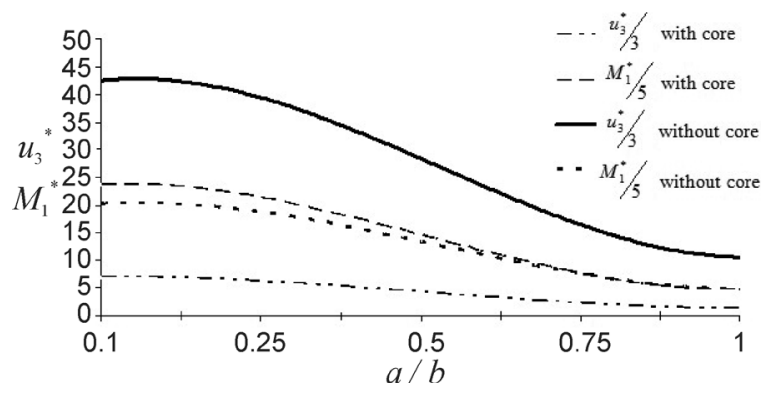

Fig. 8. Variation of normalized deflection $u_{3}^{*}$ and moment $M_{1}^{*}$ with the $a / b$ ratio

The effect of varying the laminates thickness (Fig. 9) and the core material thickness (Fig. 10) ratios are investigated by analysing the doubly-curved shell centre normalized deflections.

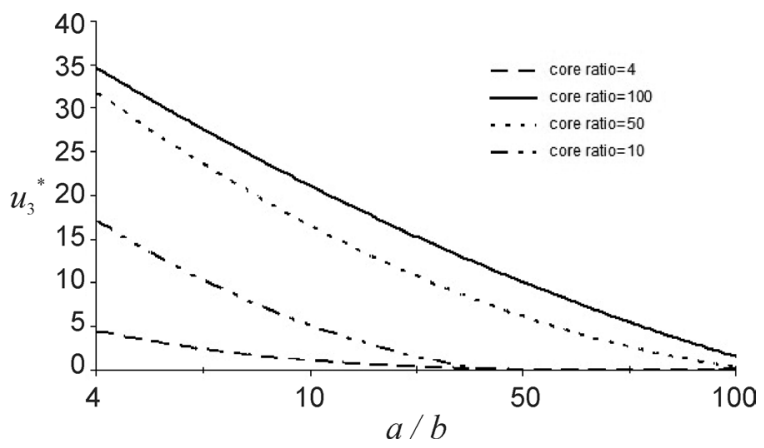

Fig. 9. Variation of central normalized deflection $u_{3}^{*}$ at varying face sheets thickness with different core thicknesses

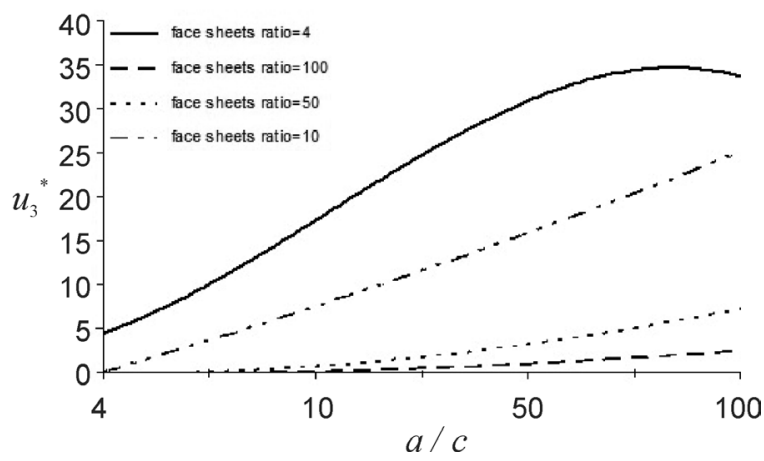

Fig. 10. Variation of central normalized deflection $u_{3}^{*}$ at varying core material thickness with different face sheets thicknesses

The tank geometry curvature, in the vertical and horizontal inclination, is described by the shallowness ratio $R / L$. Referring to this ratio, the doubly curved panel has two radii $R_{1}$ and $R_{2}$, as presented in Fig. 3. In the case of a spherical tank, the radii are made equal $R_{1}=R_{2}$, while a cylindrical tank is obtained by letting $R_{1} \rightarrow \infty$ or $R_{2} \rightarrow \infty$.

Setting both radii equal to infinity a flat plate is obtained. The effect of the shallowness ratio is presented in Fig. 11, which showing the normalized central deformations at varying $R_{1} / L$ values for spherical and cylindrical shells.

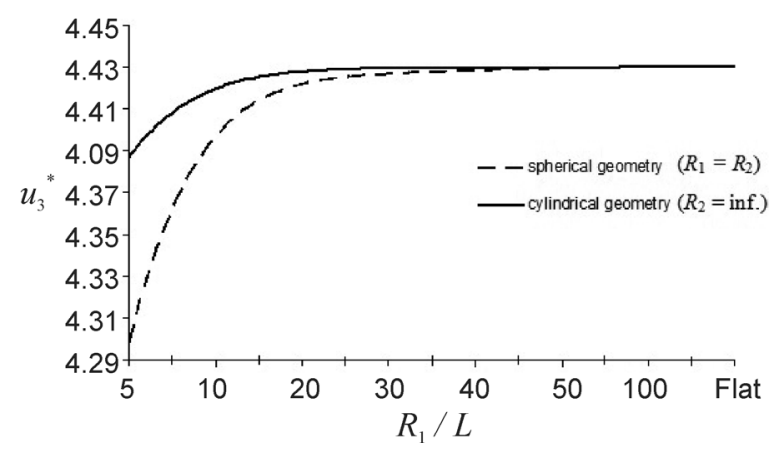

Fig. 11. Variation of central normalized deflection $u_{3}^{*}$ at varying shallowness ratios for spherical and cylindrical shells

Fig. 12 shows the inter-laminar normalized stress values for different core thicknesses; for a moderately thick $a / h=10$ symmetric $\left[0^{\circ} / 90^{\circ} /\right.$ core $\left./ 90^{\circ} / 0^{\circ}\right]$ shell $R / L=10$. While normalized stress reaches its maximum value at the top and bottom surfaces, the effect of the core material thickness is observed at the mid-surface of the shell.

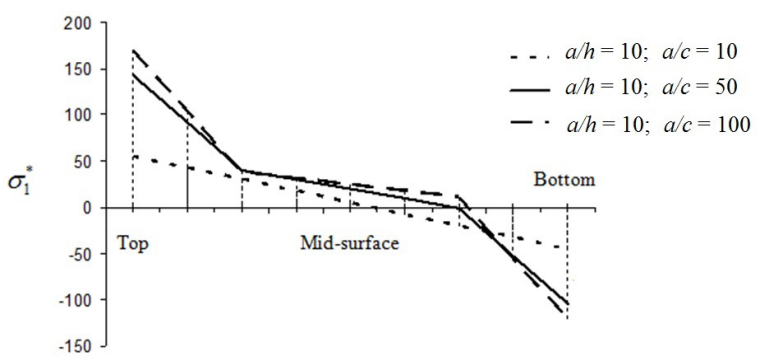

Fig. 12. Effect of core thickness by means of normalized inter-laminar stress $\sigma_{1}^{*}$ distribution at different core thicknesses

\section{CONCLUSIONS}

An analytical solution to the problem of deformation of a finite-dimensional, cross-ply, thick, doubly curved sandwich shell is applied to fully pressurized composite LPG tanks. The mathematical model of the problem is solved using the boundary discontinuous generalized double Fourier series approach, and the results are in accordance with FSDT and FEA counterparts. Therefore, the presented analytical 
method provides a benchmark solution for cylindrical and spherical fully pressurized composite LPG tanks. Further results are concluded as follows:

- The predictive assessment of the developed solution methodology is shown by comparing the numerical application results of the FSDT and FEA solutions.

- Although FEA is widely used by various researchers, the present methodology, developed for cylindrical and spherical sandwich shells may be preferred due to its lower computational demand.

- The effect of core layer thickness on the normalized central deflection of the shell is remarkable. Therefore, core layer thickness, as one of the major parameters, can be used for varying LPG tank geometries.

- The effect of geometry, as another main parameter for tank design, has major effects on deflection values. Moreover, the curvature of the shell results in moderate changes on a central deflection.

- The effect of core layer thickness over the inter-laminar stress distribution is also impressive. Increased thicknesses result in a notable decrease on inter-laminar stress distribution.

\section{ACKNOWLEDGEMENT}

CAD model of LNG carrier that is used in the article for demonstration purposes (Fig.2) is provided by SEFT Ship Design, a leading company on maritime and naval design programs in Turkey. The author would like to express a deep sense of appreciation and gratitude to General Manager Mr Semih Zorlu for his kind cooperation and help.

\section{REFERENCES}

[1] U.S. Energy Administration Annual Energy Outlook (2014). from $h t t p: / / w w w . e i a . g o v$, accessed on 2015-01-15)

[2] Fujitani, T., Emi, H., Abe, A. (2003). Ship Design and Construction. The Society of Naval Architects and Marine Engineers, Jersey City, Chapter 32.

[3] urkish Lloyd (2014). The rules of Turkish Lloyd, from http:// www.turkloydu.org, accessed on 2015-01-15.

[4] Seft (2014). SEFT Ship Design, from http://www.seft.com.tr, accessed on 2015-01-15.

[5] Mouritz, A.P., Gellert, E., Burchill, P., Challis, K. (2001). Review of advanced composite structures for naval ships and submarines. Composite Structures, vol. 53, no. 1, p. 21-41, DOI:10.1016/S0263-8223(00)00175-6.

[6] Kabir, H.R.H., Al-Khaleefi, A.M., Chaudhuri, R.A. (2001). Free vibration analysis of thin arbitrarily laminated anisotropic plates using boundary-continuous displacement Fourier approach. Composite Structures, vol. 53, no. 4, p. 469-476, DOI:10.1016/S0263-8223(01)00059-9.

[7] Chen, C.S. (2007). The nonlinear vibration of an initially stressed laminated plate. Composites: Part B Engineering, vol. 38, no. 4, p. 437-447, D0l:10.1016/j. compositesb.2006.09.002.

[8] Khalili, S.M.R., Davar, A., Fard, K.M. (2012). Free vibration analysis of homogeneous isotropic circular cylindrical shells based on a new three-dimensional refined higher order theory. International Journal of Mechanical Sciences, vol. 56, no. 1, p. 1-25, D0I:10.1016/j.jmecsci.2011.11.002.

[9] Youssif, Y.G. (2009). Non-linear design and control optimization of composite laminated doubly curved shell. Composite Structures, vol. 88, no. 3, p. 468-480, D0l:10.1016/j.compstruct.2008.05.020.

[10] Chaudhuri, R.A. (2002). On the roles of complementary and admissible boundary constraints in Fourier solutions to boundary-value problems of completely coupled rth order PDEs. Journal of Sound and Vibration, vol. 251, no. 2, p. 261313, D0l:10.1006/jsvi.2001.3913.

[11] Oktem A.S., Chaudhuri, R.A. (2008). Effect of inplane boundary constraints on the response of thick general (unsymmetric) cross ply laminates. Composite Structures, vol. 83, no. 1, p. 1-12, D0I:10.1016/j.compstruct.2007.03.002.

[12] Reddy, J.N. (2003). Mechanics of Laminated Composite Plates and Shells: Theory and Analysis, $2^{\text {nd }}$ ed. CRC Press, Boca Raton.

\section{APPENDIX A}

Definitions of $\left[K_{i j}\right]$ :

$$
\begin{aligned}
K_{11}= & A_{11} \frac{\partial^{2}}{\partial \xi_{1}^{2}}+A_{66} \frac{\partial^{2}}{\partial \xi_{2}^{2}}, \\
K_{12}= & \left(A_{12}+A_{66}\right) \frac{\partial^{2}}{\partial \xi_{1} \partial \xi_{2}}, \\
K_{13}= & \left(\frac{A_{11}}{R_{1}}+\frac{A_{12}}{R_{2}}\right) \frac{\partial}{\partial \xi_{1}}- \\
& -c_{1} E_{11} \frac{\partial^{3}}{\partial \xi_{1}^{3}}-\left(2 c_{1} E_{66}+c_{1} E_{12}\right) \frac{\partial^{3}}{\partial \xi_{1} \partial \xi_{2}^{2}}, \\
K_{14}= & \left(B_{11}-c_{1} E_{11}\right) \frac{\partial^{2}}{\partial \xi_{1}^{2}}+\left(B_{66}-c_{1} E_{66}\right) \frac{\partial^{2}}{\partial \xi_{2}^{2}}, \\
K_{15}= & \left(B_{12}-c_{1} E_{12}+B_{66}-c_{1} E_{66}\right) \frac{\partial^{2}}{\partial \xi_{1} \partial \xi_{2}}, \\
K_{22}= & A_{66} \frac{\partial^{2}}{\partial \xi_{1}^{2}}+A_{22} \frac{\partial^{2}}{\partial \xi_{2}^{2}},
\end{aligned}
$$




$$
\begin{aligned}
K_{23} & =\left(\frac{A_{12}}{R_{1}}+\frac{A_{22}}{R_{2}}\right) \frac{\partial}{\partial \xi_{2}}- \\
& -c_{1} E_{22} \frac{\partial^{3}}{\partial \xi_{2}^{3}}-\left(2 c_{1} E_{66}+c_{1} E_{12}\right) \frac{\partial^{3}}{\partial \xi_{1}^{2} \partial \xi_{2}},
\end{aligned}
$$$$
K_{24}=\left(B_{12}-c_{1} E_{12}+B_{66}-c_{1} E_{66}\right) \frac{\partial^{2}}{\partial \xi_{1} \partial \xi_{2}},
$$$$
K_{25}=\left(B_{66}-c_{1} E_{66}\right) \frac{\partial^{2}}{\partial \xi_{1}^{2}}+\left(B_{22}-c_{1} E_{22}\right) \frac{\partial^{2}}{\partial \xi_{2}^{2}},
$$$$
K_{33}=\left[\begin{array}{l}
A_{55}-6 c_{1} D_{55}+9 c_{1}^{2} F_{55}+ \\
c_{1}\left(\frac{E_{12}}{R_{1}}+\frac{E_{22}}{R_{2}}\right)+c_{1}\left(\frac{E_{11}}{R_{1}}+\frac{E_{12}}{R_{2}}\right)
\end{array}\right] \frac{\partial^{2}}{\partial \xi_{1}^{2}}+
$$$$
+\left[A_{44}-6 c_{1} D_{44}+9 c_{1}^{2} F_{44}+2 c_{1}\left(\frac{E_{12}}{R_{1}}+\frac{E_{22}}{R_{2}}\right)\right] \frac{\partial^{2}}{\partial \xi_{2}^{2}}-
$$$$
-9 c_{1}^{2} H_{11} \frac{\partial^{4}}{\partial \xi_{1}^{4}}-2 c_{1}^{2}\left(H_{12}+2 H_{66}\right) \frac{\partial^{4}}{\partial \xi_{1}^{2} \partial \xi_{2}^{2}}-
$$$$
-c_{1}^{2} H_{22} \frac{\partial^{4}}{\partial \xi_{2}^{4}}-\left[\left(\frac{A_{11}}{R_{1}^{2}}+\frac{A_{12}}{R_{1} R_{2}}\right)+\left(\frac{A_{12}}{R_{1} R_{2}}+\frac{A_{22}}{R_{2}^{2}}\right)\right] \square
$$$$
K_{35}=\left[\begin{array}{l}
A_{44}-6 c_{1} D_{44}+9 c_{1}^{2} F_{44}- \\
\frac{1}{R_{1}}\left(B_{12}-c_{1} E_{12}\right)-\frac{1}{R_{2}}\left(B_{22}-c_{1} E_{22}\right)
\end{array}\right] \frac{\partial}{\partial \xi_{2}}+
$$$$
+c_{1}\left(F_{22}-c_{1} H_{22}\right) \frac{\partial^{3}}{\partial \xi_{2}^{3}}+
$$$$
+\left[c_{1}\left(F_{12}-c_{1} H_{12}\right)+2 c_{1}\left(F_{66}-c_{1} H_{66}\right)\right] \frac{\partial^{3}}{\partial \xi_{1}^{2} \partial \xi_{2}},
$$$$
K_{35}=\left[\begin{array}{l}
A_{44}-6 c_{1} D_{44}+9 c_{1}^{2} F_{44}- \\
\frac{1}{R_{1}}\left(B_{12}-c_{1} E_{12}\right)-\frac{1}{R_{2}}\left(B_{22}-c_{1} E_{22}\right)
\end{array}\right] \frac{\partial}{\partial \xi_{2}}+
$$$$
+c_{1}\left(F_{22}-c_{1} H_{22}\right) \frac{\partial^{3}}{\partial \xi_{2}^{3}}+
$$$$
+\left[c_{1}\left(F_{12}-c_{1} H_{12}\right)+2 c_{1}\left(F_{66}-c_{1} H_{66}\right)\right] \frac{\partial^{3}}{\partial \xi_{1}^{2} \partial \xi_{2}}
$$

$$
\begin{aligned}
K_{44}= & {\left[D_{11}-2 c_{1} F_{11}+c_{1}^{2} H_{11}\right] \frac{\partial^{2}}{\partial \xi_{1}^{2}}+} \\
& +\left[D_{66}-2 c_{1} F_{66}+c_{1}^{2} H_{66}\right] \frac{\partial^{2}}{\partial \xi_{2}^{2}}- \\
& -\left(A_{55}+6 c_{1} D_{55}+9 c_{1}^{2} F_{55}\right), \\
K_{45}= & {\left[\begin{array}{l}
D_{12}-c_{1} F_{12}+D_{66}-c_{1} F_{66}- \\
c_{1}\left(F_{12}-c_{1} H_{12}\right)-c_{1}\left(F_{66}-c_{1} H_{66}\right)
\end{array}\right] \frac{\partial^{2}}{\partial \xi_{1} \partial \xi_{2}}, } \\
K_{55}= & {\left[D_{66}-2 c_{1} F_{66}+c_{1}^{2} H_{66}\right] \frac{\partial^{2}}{\partial \xi_{1}^{2}}+} \\
+ & {\left[D_{22}-2 c_{1} F_{22}+c_{1}^{2} H_{22}\right] \frac{\partial^{2}}{\partial \xi_{2}^{2}}-} \\
& -A_{44}+3 c_{1} D_{44}+3 c_{1}\left(D_{44}-3 c_{1} F_{44}\right),
\end{aligned}
$$

where;

$$
\begin{aligned}
& \left(A_{i j}, B_{i j}, D_{i j}\right)=\sum_{k=1}^{N} \int_{\xi_{k-1}}^{\xi_{k}} Q_{i j}^{(k)}\left(1, z, z^{2}\right) d z, \\
& \left(E_{i j}, F_{i j}, H_{i j}\right)=\sum_{k=1}^{N} \int_{\xi_{k-1}}^{\xi_{k}} Q_{i j}^{(k)}\left(z^{3}, z^{4}, z^{6}\right) d z, \\
& c_{1}=-\frac{4}{3 h^{2}} .
\end{aligned}
$$

\section{APPENDIX B}

Definition of boundary Fourier coefficients $\overline{a_{n}}=\frac{4}{a b} \int_{0}^{b}\left[u_{1}\left(a, \xi_{2}\right)-u_{1}\left(0, \xi_{2}\right)\right] \cos \left(\beta \xi_{2}\right) d \xi_{2}$,

$\overline{b_{n}}=-\frac{4}{a b} \int_{0}^{b}\left[u_{1}\left(a, \xi_{2}\right)-u_{1}\left(0, \xi_{2}\right)\right] \cos \left(\beta \xi_{2}\right) d \xi_{2}$,

$\overline{c_{m}}=\frac{4}{a b} \int_{0}^{a}\left[u_{2,2}\left(\xi_{1}, b\right)-u_{2,2}\left(\xi_{1}, 0\right)\right] \sin \left(\alpha \xi_{1}\right) d \xi_{1}$,

$\overline{d_{m}}=-\frac{4}{a b} \int_{0}^{a}\left[u_{2,2}\left(\xi_{1}, b\right)+u_{2,2}\left(\xi_{1}, 0\right)\right] \sin \left(\alpha \xi_{1}\right) d \xi_{1}$. 